\title{
Phase transitions and correlations in fracture processes where disorder and stress compete
}

\author{
Santanu Sinha, ${ }^{1,2, *}$ Subhadeep Roy, ${ }^{2, \dagger}$ and Alex Hansen ${ }^{2,1, \hbar}$ \\ ${ }^{1}$ Beijing Computational Science Research Center, 10 East Xibeiwang Road, Haidian District, Beijing 100193, China \\ ${ }^{2}$ PoreLab, Department of Physics, Norwegian University of Science and Technology, NO-7491 Trondheim, Norway
}

(Received 16 June 2020; accepted 29 September 2020; published 20 October 2020)

\begin{abstract}
We study the effect of the competition between disorder and stress enhancement in fracture processes using the local load sharing fiber bundle model, a model that hovers on the border between analytical tractability and numerical accessibility. We implement a disorder distribution with one adjustable parameter. The model undergoes a localization transition as a function of this parameter. We identify an order parameter for this transition and find that the system is in the localized phase over a finite range of values of the parameter bounded by a transition to the nonlocalized phase on both sides. The transition is of first order at one side and of second order at the other. The critical exponents characterizing the second-order transition are close to those characterizing the percolation transition. We determine the spatiotemporal correlation function in the localized phase. It is characterized by two power laws as in invasion percolation. We find exponents that are consistent with the values found in that problem.
\end{abstract}

DOI: 10.1103/PhysRevResearch.2.043108

\section{INTRODUCTION}

It has been known for a long time that heterogeneities make materials more resilient against failure under load by offsetting the point at which a microfracture becomes unstable [1]. Examples of materials where heterogeneities play an important role are concrete [2,3] and carbon-fiber composites [4] which possess larger strength and toughness than the individual components [5,6]. Heterogeneities introduce spatial disorder in the local material strength and create a spatially dependent dynamic stress field.

The interplay between disorder and dynamical effects during the breakdown process of brittle materials was the focus of much research within the statistical physics community during the 1980s and 1990s [7]. Those studies were based on a number of lattice-based models where the links would have a maximum load before they would fail drawn from some spatially uncorrelated distribution [8-13].

We summarize the picture that emerged qualitatively in the following. There are two reasons for a failure to appear locally in a disordered material. Either it is due to the material being weak at that local point or it is due to the stress being high there. Imagine now loading the disordered material. At the beginning of the breakdown process, the material will fail where it is weak as there are no-or few-points with

\footnotetext{
*santanu@csrc.ac.cn

${ }^{\dagger}$ subhadeep.roy@ntnu.no

¥alex.hansen@ntnu.no
}

Published by the American Physical Society under the terms of the Creative Commons Attribution 4.0 International license. Further distribution of this work must maintain attribution to the author(s) and the published article's title, journal citation, and DOI. high stress. When local failures develop, spots with intense stress appear at the tips of microcracks. As the failure process proceeds, these high-stress spots will start dominating. As it was argued by Roux and Hansen [14] disorder makes local failures repulsive whereas the stress field makes them attractive. Imagine that there is a first local failure. Draw a sphere around this first local failure and identify the weakest spot within the sphere. The larger the sphere is, the weaker the weakest spot within it will be. As a result, if the next local failure is due to the local weakness of the material, it will occur as far as possible from the first one. Hence, the failures are repulsive when they are caused by the disorder in the material strength. The effect of the stress field is opposite. The closer one is to a local failure, the higher the largest stress will be. This makes it more likely that the next failure will be close to the one that just appeared. Hence, local failures attract each other when they are due to high stress field. The result of this is that there is a competition between disorder and stress concentration throughout the failure process. Early in the failure process, the disorder tends to dominate, resulting in local failures appearing distributed throughout the material. However, as the stress field takes over, there is localization ending with a single growing crack beginning to dominate. Depending on the disorder, localization may occur early or later in the process: The wider the disorder, the later on the onset of localization would occur. In the limit of infinite disorder [15-17], localization never sets in, and the failure process is a screened percolation-i.e., a process where links fail at random given that they are connected in such a way that they carry stress. When the disorder is weak enough to cause a competition with the stresses, a phase diagram may be constructed showing the onset of localization as a function of the disorder [18-20].

It is the aim of this paper to study the effect of the competition between disorder and stress enhancement using the fiber 
bundle model [21,22]. The advantage of using this model over other models is that it is not computationally demanding, leading to good statistics for large samples. Furthermore, it has a high level of analytical tractability. We describe the fiber bundle model in detail in Sec. II. It should be noted that Stormo et al. [23] studied the interplay between disorder and stress enhancement in the soft clamp model which is a more complex version of the fiber bundle model than the version we study here. Their conclusions differ from those we present here; this is due to a very different way of analyzing the fracture process. We will discuss it in the concluding section. We implement a disorder distribution that has one adjustable parameter. By tuning this parameter, we produce disorders with power law tails towards either zero strength or infinite strength. We focus in Sec. III on the localization transition that occurs for some value of the disorder parameter, see Fig. 2. For the range of the values of the disorder parameter that produces power-law tails towards zero strength, we find a second-order phase transition, whereas for the range of values for the disorder parameter that produces a power-law tail towards infinite strength, we find a first-order phase transition. We determine the values of critical exponents associated with the second-order phase transition. They are close to those found in percolation. In Sec. IV, we study the spatiotemporal correlation function first introduced by Furuberg et al. [24] in connection with invasion percolation. We determine the scaling exponents characterizing the correlation function and find them to be close to the values observed for invasion percolation. We relate the exponents to other exponents characterizing the transition using theory developed by Roux and Guyon [25], Maslov [26], and Gouyet [27]. We end by drawing our conclusions in Sec. V.

\section{DESCRIPTION OF THE MODEL}

The fiber bundle model is a model of fracture where one can control the range of stress enhancement upon the appearance of a crack. In this model, $N$ fibers (Hookean springs) are placed between two clamps under external force $F$. Each fiber carries a force,

$$
f=\kappa x,
$$

where $\kappa$ and $x$, respectively, are the elastic constant and extension of the fiber. The extension of each fiber has a threshold $\left(x_{t}\right)$, beyond which it fails, and the load it was carrying is distributed among surviving fibers according to some preset rule. The stress distribution scheme models the local stress enhancement whereas the disorder in $x_{t}$ models the local material heterogeneity. If the load of the failed fibers is distributed over all the surviving fibers, there is no local stress enhancement. This is the equal load sharing (ELS) scheme. The ELS fiber bundle model was introduced by Peirce in 1926 [28] as a simple model for failure in fibrous materials. Daniels approached the ELS fiber bundle model as a problem in statistics in a seminal paper in 1945 [29]. If the load is distributed only to the nearby fibers, we are dealing with the local load sharing (LLS) model [30,31]. Here, local stress enhancement competes with the local heterogeneity leading to localization. Sornette introduced the ELS fiber bundle model to the statistical physics community in 1992 [32]. Soon, the focus of this community was on the rich avalanche statistics that this model offers [33-36], which, in the ELS case, is analytically tractable.

The sequential-or time-correlation between failure events in the fiber bundle model makes it possible to explore the brittle to ductile transition [37] within it, a well-studied phenomenon in material science. The spatial correlations between the failures, on the other hand, have not been studied in detail.

In the LLS fiber bundle model, the load carried by the failed fibers is distributed equally among their nearest-intact neighbors. We define a crack as a cluster of $s$ failed nearestneighbor fibers. The perimeter of the crack is the set of $p$ intact fibers that are nearest neighbors to the failed fibers constituting the crack. These nearest neighbors define the hull of the cluster [38]. The force on an intact fiber $i$ at any instance is then given by

$$
f_{i}=\sigma\left(1+\sum_{J(i)} \frac{s_{J(i)}}{p_{J(i)}}\right),
$$

where $\sigma=F / N$, the force per fiber. The summation runs over all cracks $J(i)$ that are neighbors to fiber $i$. This redistribution scheme is independent of the lattice topology and also independent of the failure history [39], which means, the complete stress field can be calculated from the present arrangement of intact and broken fibers without having to take into account the order in which the fibers failed.

Here, we have adopted a quasistatic failure process where, at each time step, one single fiber breaks. This is equivalent to the hypothetical experimental setup where the external stress is increased from zero until the first fiber that reaches the equality $f_{i}=\kappa x_{t, i}$ breaks, and, then, the external stress immediately reduced to zero. At the next time step, the stress is increased again to break the next fiber and then reduced to zero again. This algorithm provides the complete order of the fibers that would break during the failure event. In such a process, a bundle with $N$ fibers will break in $N$ time steps. Now, suppose $t$ fibers have failed in $t$ steps. We determine which fiber will fail at time $t+1$ in the following way [22]. Let $f_{i}^{1}$ be the force on fiber $i$ if we set the average force on the fibers $\sigma=1$. We, then, calculate

$$
\lambda(t+1)=\max _{i}\left(\frac{f_{i}^{1}}{\kappa x_{t, i}}\right),
$$

which denotes the fiber $i(t+1)$ that fails at time $t+1$. The force $\sigma=\sigma(t+1)$ at which this fiber fails is given by

$$
\sigma(t+1)=\frac{1}{\lambda(t+1)} .
$$

The failure thresholds $x_{t}$ of the fibers are assigned by generating a random number over the unit interval and raising it to power $D$ which corresponds to the cumulative distribution $[13,20]$,

$$
P\left(x_{\mathrm{t}}\right)=\left\{\begin{array}{lll}
x_{\mathrm{t}}^{1 /|D|}, & x_{t} \in[0,1], & \text { when } D>0, \\
1-x_{\mathrm{t}}^{-1 /|D|}, & x_{t} \in[1, \infty), & \text { when } D<0 .
\end{array}\right.
$$

This threshold distribution allows us to control the disorder by the value of $D$, a higher value of $|D|$ implies higher disorder. Furthermore, $D>0$ and $D<0$, respectively, correspond to 

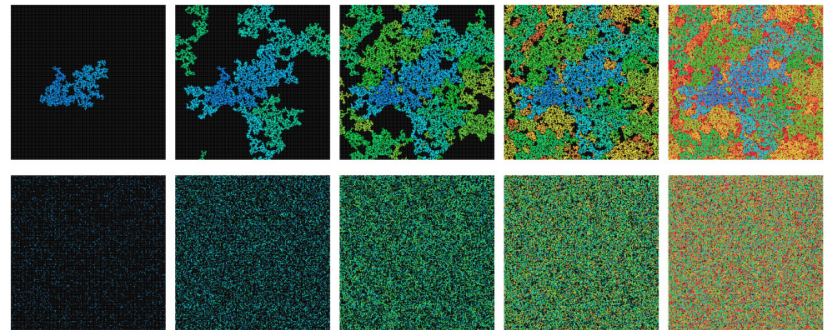

.

$D>0$
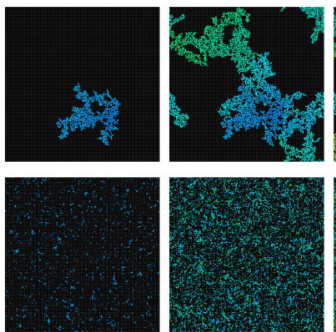
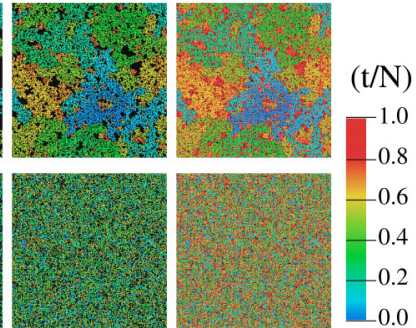

$D<0$

FIG. 1. Growth of cracks in a bundle of $256 \times 256$ fibers with threshold distribution from Eq. (5). The left and right panels correspond to $D>0$ and $D<0$, respectively. The top and bottom rows in each panel correspond to low $(|D|=0.02)$ and high $(|D|=2.0)$ disorders, respectively. The black sites are intact fibers, and the colored sites are broken fibers. The colors represent the breaking sequence $(\tau=t / N)$ as indicated by the scale at right. The five snapshots in each set show the evolution of cracks at $\tau=0.04,0.2,0.4,0.6$, and 0.9 .

the distributions with power-law tails towards weaker and stronger fibers, which, as we will see, make the failure dynamics very different. It might seem overly restrictive to limit the threshold distributions to power laws either towards zero or infinity as there are many other classes of distributions, e.g., exponentials. However, the fracture process is controlled by the tails of the threshold distributions, either towards zero or infinity. In finite-size systems where there is a finite number of thresholds, these tails turn into the extreme value distributions, of which there are only three: Weibull, Fréchet, and Gumbel [40]. Power-law tails towards zero, including distributions that approach a constant at zero, produce the Weibull extreme distribution, and power-law tails towards infinity produce the Fréchet extreme distribution. Distributions that fall off exponentially or faster produce the Gumbel extreme distribution. This latter distribution, however, falls off so quickly (the exponential of an exponential), that it essentially behaves as no disorder at all. Hence, our choice, Eq. (5), essentially exhausts the possibilities. We do, however, exclude the possibility to have a power-law tail towards zero and infinity simultaneously.

\section{LOCALIZATION TRANSITION}

We implement the model on a square lattice of size $N=$ $L \times L$ fibers. The growth of cracks for $L=256$ at different disorders are shown in Fig. 1 where we see two distinct regimes, a single crack growth at low disorder (top row) and random failures at high disorder (bottom row). The color scale in this figure represents where in the breaking sequence a given fiber fails, parametrized by $\tau=t / N$, which we will discuss in detail later. At very low disorder, the stress enhancement at the crack perimeter wins over the fiber strengths and the crack always grows from the perimeter of the existing crack. The local disorder at the perimeter makes the crack grow as an invasion percolation cluster [41].

At high disorder, the strength of fibers win over the stress enhancement, and we see random clusters of failed fibers appear. These two cases appear to be same for both $D>0$ and $D<0$, however, a completely different picture emerges between these two regimes when we observe crack growth at intermediate disorders. In Fig. 2, we plot the crack density $n_{c}=N_{c} / N$, where $N_{c}$ is the number of cracks (clusters) when $t$ fibers have broken for different disorders as a function of $\tau=t / N$. We see that $n_{c}$ stays close to $0\left(N_{c}=1\right)$ for the whole failure process for small $|D|$ values whereas for large $|D|, n_{c}$ increases with $\tau$ and reaches a maximum $\left(n_{c}^{\max }\right)$, be-
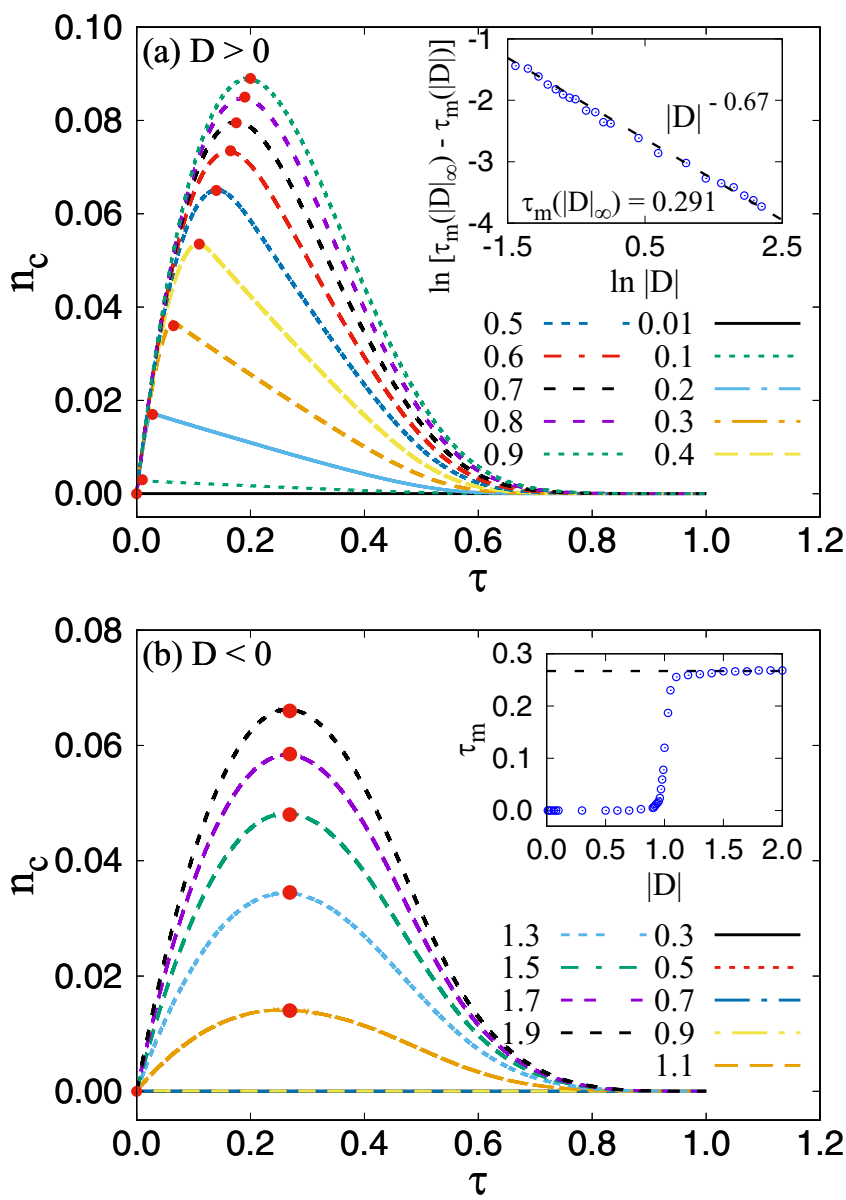

FIG. 2. Variation of crack densities $n_{c}$ with fraction of broken bonds $\tau$ for $L=256$ at different disorders. The $|D|$ values are indicated in the legends. The red dots indicate the peaks at $\left(\tau_{\mathrm{m}}, n_{c}^{\max }\right)$. For $D>0$, we see a gradual increase in $\tau_{\mathrm{m}}$ with increasing $|D|$ whereas for $D<0$, we first see a discontinuity from zero to a finite value and, then, it remains almost constant at $\approx 0.27$. The inset of (a) shows the scaling $\tau_{m}^{+}\left(|D|_{\infty}\right)-\tau_{m}^{+}(|D|) \sim|D|^{-\gamma}$ for $D>0$ where $\gamma=0.67 \pm 0.01$ and $\tau_{m}^{+}\left(|D|_{\infty}\right)=0.291 \pm 0.011$. The inset of (b) shows the convergence of $\tau_{m}^{-}$towards $|D| \rightarrow \infty$ for $D<0$. 


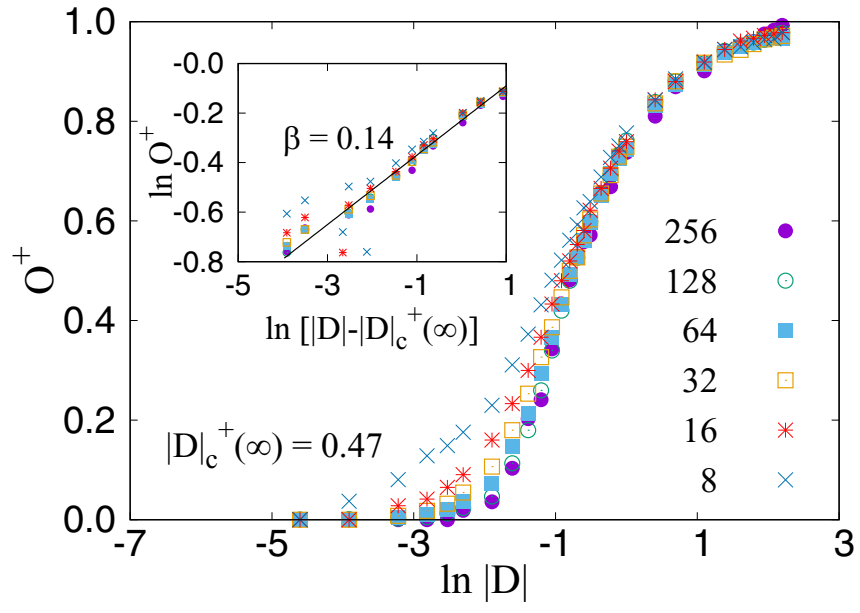

FIG. 3. Plot of order parameter $O^{+}$as a function of disorder $|D|$ for $D>0$. Different symbols indicate different system sizes $L$ where data are averaged over 200 to 100000 samples depending on $L$. In the inset, we plot the scaling relation given in Eq. (8) where we find $|D|_{\mathrm{c}}^{+}(\infty)=0.47 \pm 0.04$ and $\beta=0.14 \pm 0.05$.

yond which, the cracks start to coalesce as more fibers are broken. The position of the peaks at the $\tau$ axis, marked by the red dots in Fig. 2, vary continuously with disorder for $D>0$ whereas the peak stays at almost same position for $D<0$ after a discontinuity between the low and high $|D|$ values. Moreover, for two disorders with same values of $n_{c}^{\max }$, the peak appears much earlier for $D>0$ compared to $D<0$. This shows that individual cracks appear randomly for $D>0$ whereas cracks grow in size together with the appearance of new cracks for $D<0$. Intuitively, when the threshold distribution has a power-law tail towards strong bonds $(D<0)$, probability to find weak bonds at existing crack perimeters are higher, which makes existing cracks to grow. Whereas when the power-law tail is towards the weak bonds $(D>0)$, the probability to find strong bonds at the perimeter is high, and new cracks appear at different positions than the perimeter.

To characterize this localization transition, we define an order parameter,

$$
O^{-/+}(|D|, L)=\frac{\tau_{\mathrm{m}}^{-/+}(|D|, L)}{\tau_{\mathrm{m}}^{-/+}\left(|D|_{\infty}, L\right)},
$$

where the plus and minus signs correspond to $D>0$ and $D<0$, respectively. Here, $\tau_{\mathrm{m}}^{-/+}(|D|, L)$ is the fraction of broken fibers at $n_{c}=n_{c}^{\max }$ and $\tau_{\mathrm{m}}^{-/+}\left(|D|_{\infty}, L\right)$ is the value of $\tau_{\mathrm{m}}$ for $|D| \rightarrow \infty$, i.e., when the breakdown is an uncorrelated percolation process. The measurements of $\tau_{\mathrm{m}}^{-/+}\left(|D|_{\infty}, L\right)$ for $L=256$ are shown in the insets of Fig. 2. For $D>0$, we observe the scaling,

$$
\tau_{\mathrm{m}}^{+}\left(|D|_{\infty}\right)-\tau_{\mathrm{m}}^{+}(|D|) \sim|D|^{-\gamma},
$$

where we have that $\gamma=0.67 \pm 0.01$ and $\tau_{\mathrm{m}}^{+}\left(|D|_{\infty}\right)=$ $0.291 \pm 0.011$. For $D<0, \tau_{m}^{-}(|D|)$ converges to a maximum value more abruptly from which we find $\tau_{\mathrm{m}}^{-}\left(|D|_{\infty}\right)=0.27$ as shown by the dashed line in the inset.

The transition for $D>0$ is explored in Fig. 3 where we plot $O^{+}(|D|, L)$ as a function of disorder for different system sizes. Here, $O^{+}$varies continuously from 1 to 0 similar to a
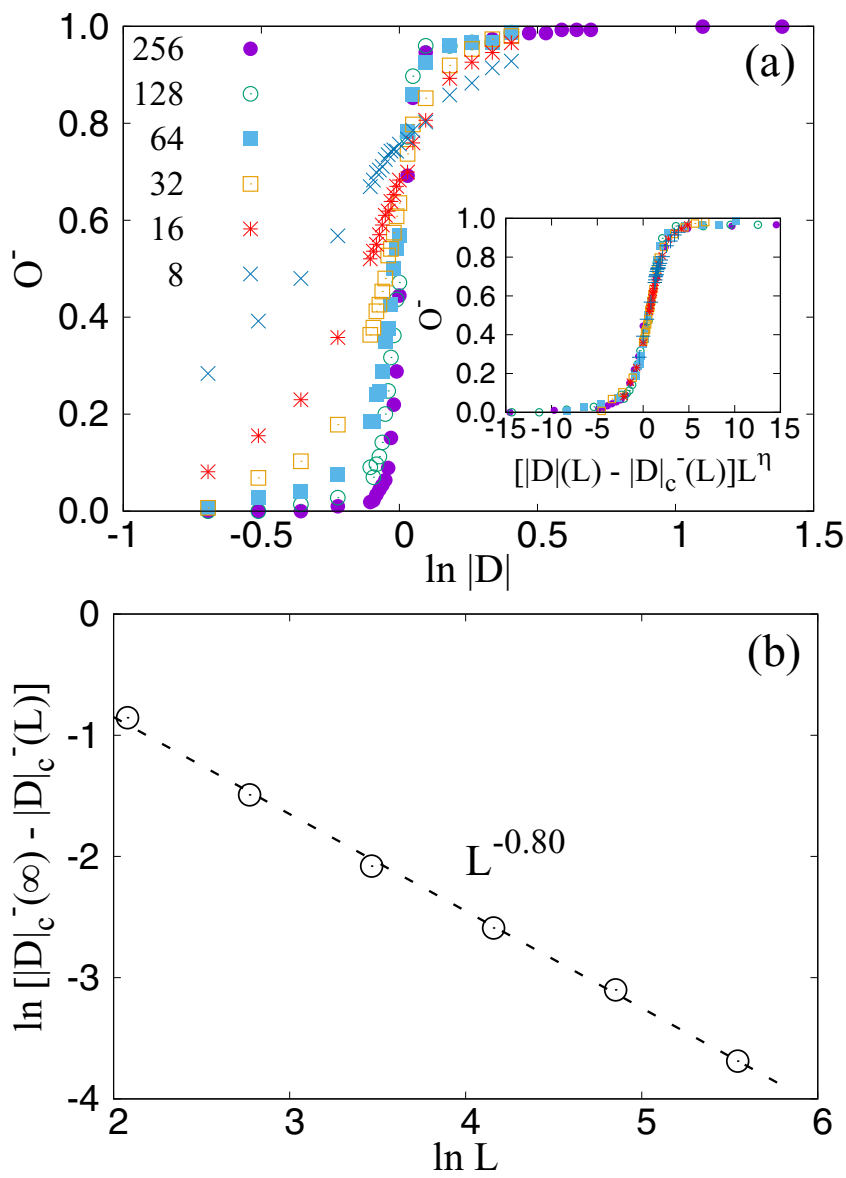

FIG. 4. Plot of order parameter $O^{-}$as a function of disorder for $D<0$ is plotted in (a) which shows abrupt transition with the increase in system size $L$. The inset in (a) shows the scaling with different $L$ 's according to Eq. (9). In (b), we plot $|D|_{\mathrm{c}}^{-}(\infty)-|D|_{\mathrm{c}}^{-}(L) \sim$ $L^{-\theta}$ where we find $|D|_{\mathrm{c}}^{-}(\infty)=1.03 \pm 0.02$ with $\theta=0.80 \pm 0.01$.

second-order phase transition. We find the following scaling for $O^{+}$:

$$
O^{+}(|D|, L) \sim\left[|D|(L)-|D|_{\mathrm{c}}^{+}(\infty)\right]^{\beta},
$$

where $|D|_{\mathrm{c}}^{+}(\infty)=0.47 \pm 0.04$ and $\beta \approx 0.14 \pm 0.05$. The exponent $\beta$ is close to $5 / 36$, the order parameter exponent for percolation in two dimensions $[42,43]$. However, $1 / 8$, the exact value from the Onsager solution of the two-dimensional Ising model [44] is also within the precision with which we know $\beta$.

The transition for $D<0$ is presented in Fig. 4 where we plot $O^{-}(|D|, L)$ as a function of disorder. Similar to a firstorder phase transition, $O^{-}$shows sharp transition from 0 to 1 as the system size $L$ is increased. The plots for different $L$ 's can be scaled as [45]

$$
O^{-}(|D|, L) \sim \Phi\left\{\left[|D|(L)-|D|_{\mathrm{c}}^{-}(L)\right] L^{\eta}\right\},
$$

with $\eta=0.7$. This is shown in the inset of Fig. 4(a). The transition disorder $|D|_{\mathrm{c}}^{-}(L)$ is observed to be a decreasing function of $L$. To find the value of $|D|_{\mathrm{c}}^{-}$as $L \rightarrow \infty$, we use the scaling $|D|_{\mathrm{c}}^{-}(\infty)-|D|_{\mathrm{c}}^{-}(L) \sim L^{-\theta}$ with $|D|_{\mathrm{c}}^{-}(\infty)=1.03 \pm 0.02$ and $\theta=0.80 \pm 0.01$. This is shown in Fig. 4(b). 

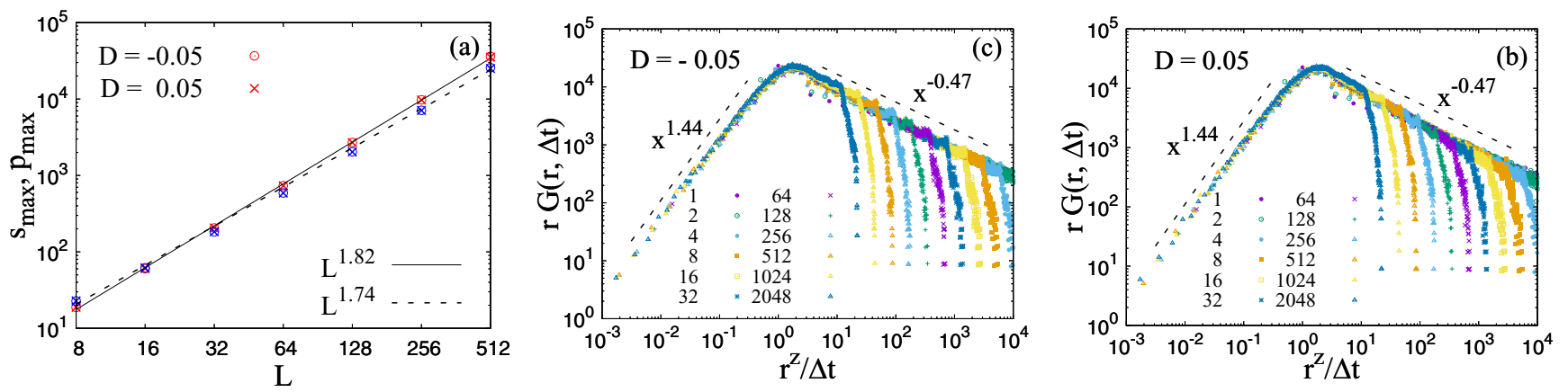

FIG. 5. (a) Plot of the largest crack size $s_{\max }$ (red symbols) and the largest perimeter size $p_{\max }$ (blue symbols) as a function of the system size $L$. From the slopes, we find $d_{\mathrm{f}}=1.82 \pm 0.02$ and $d_{\mathrm{h}}=1.74 \pm 0.02$. In (b) and (c), we plot the pair correlation function [Eq. (10)] for $D=+0.05$ and -0.05 , respectively, where we used $z=d_{\mathrm{f}}$ obtained from (a). The different plots correspond to different values of $\Delta t$ as indicated in the figures. From the two power-law scalings, we find the exponents $a=1.44 \pm 0.04$ and $b=0.47 \pm 0.06$.

\section{SPATIOTEMPORAL CORRELATIONS}

We will now explore the spatial and temporal correlations during the breakdown process. First, we measure the fractal dimension of single cracks at low disorder, defined as $s_{\max } \sim L^{d_{\mathrm{f}}}$ where $s_{\max }$ is size of the largest incipient infinite cluster. This is shown in Fig. 5(a). We find $d_{\mathrm{f}}=1.82 \pm 0.02$ which is close to the fractal dimension of invasion percolation cluster with trapping $[41,46]$. The colors in Fig. 1 represent the sequence of fiber breaking and together with the positions of broken fibers, they show the spatiotemporal map of the failure process. For random failures at high disorder (bottom row), the pixels (each representing a fiber) of different colors are randomly mixed, whereas for the single crack growth (top row), the different colors are clustered, indicating localization and, hence, spatial and temporal correlations. To quantify this correlation, we measure pair correlation function $G(r, \Delta t)$, defined as follows: If a fiber at position $\boldsymbol{r}_{\mathbf{0}}$ breaks at a time $t$, then, $G(r, \Delta t)$ provides the probability that another fiber at $\boldsymbol{r}_{1}$ will break at time $t+\Delta t$, where $r=\left|\boldsymbol{r}_{1}-\boldsymbol{r}_{0}\right|$. Here, time $t$ is measured in terms of the number of broken fibers. This correlation function was first introduced by Furuberg et al. [24] for invasion percolation. We assume in the following that $G(r, \Delta t)$ has the scaling form [24]

$$
G(r, \Delta t)=r^{-1} g\left(\frac{r^{z}}{\Delta t}\right),
$$

where $z$ is a dynamic exponent. In Fig. 5, we plot $r G(r, \Delta t)$ for the single crack growth regime as a function of $r^{z} / \Delta t$ for $D>$ 0 (b) and $D<0$ (c). For $z=d_{\mathrm{f}}$, the single-parameter function $g(u)$ shows power-law behavior in both the large- and smallargument limits,

$$
g(u) \sim \begin{cases}u^{a}, & u \ll 1 \text { (long time range) } \\ u^{-b}, & u \gg 1 \text { (short time range) }\end{cases}
$$

with a peak at $u=1$ or at $\Delta t=r^{d_{\mathrm{f}}}$ as we observe in the figure. This implies that the most probable growth of the crack after time $\Delta t$ occurs at a distance $r^{d_{\mathrm{f}}}$, or the most probable growth at a distance $r$ occurs after a time $\Delta t^{1 / d_{\mathrm{f}}}$. The exponent $a$ in the long time range is found to be $1.44 \pm 0.04$ as shown in Fig. 5. This matches the exponent for invasion percolation dynamics [24]. Recently, this spatiotemporal scaling was observed experimentally for two-phase flow in a porous medium during slow drainage [47] where the exponent $a$ was found ranging between 1.4 and 1.73. For the power law in the small-argument limit, we obtain the exponent $b=0.47 \pm$ 0.06 , which is different from 0.68 obtained by Furuberg et al. for invasion percolation dynamics [24].

The exponents and the errors mentioned above were obtained by least-squares fitting of the numerical data. For the plots with multiple data sets, such as in the Fig. 3 inset, Figs. 5(b) and 5(c), the indicated error is the average of all the errors for each individual set.

We may relate the exponents $a$ and $b$ to the exponents controlling the avalanche structure of the failure process following Roux and Guyon [25], Maslov [26], and Gouyet [27]. The minimum force applied to the fiber bundle for fiber number $t+1$ to fail is $\sigma(t+1)$ given in Eq. (4). We define a forward avalanche (or burst) of size $\Theta$ starting at time $t$ to be $[26,33]$

$$
\sigma(t+k)<\sigma(t) \text { for } k<\Theta \text { and } \sigma(t+\Theta) \geqslant \sigma(t) .
$$

A backwards avalanche (or burst) we define as

$$
\sigma(t-k)<\sigma(t) \text { for } k<\Theta \text { and } \sigma(t-\Theta) \geqslant \sigma(t)
$$

Roux and Guyon [25] define two distributions $P_{\Delta t}$ and $Q_{\Theta}(r)$. The first one $P_{\Delta t}$ gives the distribution of the smallest avalanches $\Theta$ that pass through $\sigma(t)$ and $\sigma(t-\Delta t)$ averaged over $\sigma(t)$. It obeys the power law,

$$
P_{\Delta t}(\Theta) \propto \frac{1}{\Delta t}\left(\frac{\Theta}{\Delta t}\right)^{-c_{b}} H(\Theta-\Delta t),
$$

where $H(x)$ is the Heaviside function which is one when $x>0$ and zero otherwise. The second one $Q_{\Theta}$ gives the distribution of distances between failing fibers within an avalanche of size $\Theta$. Roux and Guyon assume it to follow the power law:

$$
Q_{\Theta}(r) \propto \frac{1}{\Theta^{1 / d_{\mathrm{f}}}}\left(\frac{r}{\Theta^{1 / d_{\mathrm{f}}}}\right)^{\zeta} .
$$

The spatiotemporal correlation function $G(r, \Delta t)$ may then be constructed from these two probability distributions,

$$
G(r, \Delta t)=\int_{0}^{\infty} P_{\Delta t}(\Theta) Q_{\Theta}(r) d \Theta,
$$


which integrates to

$$
r G(r, \Delta t) \propto \begin{cases}\left(\frac{r^{d_{\mathrm{f}}}}{\Delta t}\right)^{(1+\zeta) / d_{\mathrm{f}}}, & \text { if } r^{d_{\mathrm{f}}}<\Delta t, \\ \left(\frac{r^{d_{\mathrm{f}}}}{\Delta t}\right)^{1-c_{b}}, & \text { if } r^{d_{\mathrm{f}}}>\Delta t .\end{cases}
$$

Hence, we have in Eq. (11) that $a=(1+\zeta) / d_{\mathrm{f}}$ and $b=$ $c_{b}-1$. Roux and Guyon suggest $\zeta=d_{\mathrm{f}}-1$ making $a=1$. We propose, here, that $\zeta=d_{\mathrm{h}}$, the fractal dimension of the hull [48], defined as the set of the sites that are connected to the cluster and at the neighbor to the surroundings. In the LLS fiber bundle model, the stress of a crack is re-distributed to its perimeter, the neighboring unbroken fibers of the crack. In Fig. 5, we plot this largest perimeter size $p_{\max }$ with the system size $L$, and from the slope, we find $d_{\mathrm{h}}=1.74 \pm 0.02$. This value matches with the value of $d_{\mathrm{h}}$ for percolation hull, known to be $7 / 4$ by the relation with $v$ by $d_{\mathrm{h}}=1+1 / v$, where $v$ is the correlation length exponent which is equal to $4 / 3$ in two-dimensional percolation $[49,50]$. Hence, we find that

$$
a=\frac{1+d_{\mathrm{h}}}{d_{\mathrm{f}}} .
$$

With $d_{\mathrm{f}}=1.82 \pm 0.02$ and $d_{\mathrm{h}}=1.74 \pm 0.02$, we find $a=$ $1.51 \pm 0.04$, which is close to the observed value.

Maslov [26] related the backwards avalanche exponent $c_{b}$ to the exponent $c_{f}$ that governs the probability to find a forward avalanche of size $\Theta$ when the stress is $\sigma_{0}$ via the expression,

$$
c_{b}=3-c_{f} .
$$

Furthermore, Goyuet, in turn, related $c_{f}$ to $d_{\mathrm{h}}, d_{\mathrm{f}}$, and $v$,

$$
c_{f}=1+\frac{d_{\mathrm{h}}-1 / v}{d_{\mathrm{f}}},
$$

so that

$$
b=1-\frac{d_{\mathrm{h}}-1 / v}{d_{\mathrm{f}}},
$$

which leads $b=0.46$. This is also in accordance with the value we observe $b=0.47$.

\section{CONCLUSION}

We have here studied the effect of the competition between disorder and stress enhancement using the local load sharing fiber bundle model. We have performed this by varying the disorder using a threshold distribution controlled by a single-parameter $D$. When $D$ is positive, the threshold distribution is a power law towards infinitely weak elements, and when $D$ is negative, the distribution is a power law towards infinitely strong elements. By defining an order parameter distinguishing between the localized and the nonlocalized phases, we find two phase transitions, one at $D=D_{c}^{+}=0.47 \pm 0.04$ and one at $D=D_{c}^{-}=-1.03 \pm 0.02$. The transition for $D>0$ is second order, and the transition for $D<0$ is first order. The second-order transition is governed by critical exponents that are consistent with percolation but also close to those found for the two-dimensional Ising model. We, then, studied the spatiotemporal correlation function, finding the same behavior as first seen by Furuberg et al. [24] in an invasion percolation context. Some numerical values for the exponents controlling the correlation function are different than found by Furuberg et al., which, by following the scaling analysis of Roux and Guyon [25], Maslov [26], and Gouyet [27], we successfully related to other exponents describing the geometry of the cracks.

The conclusions we present here differ from those that Stormo et al. [23] presented. In that paper, based on the softclamp fiber bundle model, the threshold distribution was the uniform one on the unit interval, i.e., $D=1$. The parameter that was varied, was the elastic constant of the clamps to which the fibers are connected. For a given value of this parameter, the breakdown process would proceed in the beginning as an uncorrelated percolation process up to a certain point at which localization would set in. From this point on, the breakdown process would continue through the growth of a single cluster of broken fibers. This transition would not be a phase transition but a crossover.

It would be of great interest to repeat the analysis we have presented here using the soft-clamp fiber bundle model by varying the disorder. Only, then, we will be able to distinguish what is model dependent in our conclusions and what is not.

\section{ACKNOWLEDGMENTS}

The authors thank E. G. Flekkøy for interesting discussions. This work was partly supported by the Research Council of Norway through its Centres of Excellence funding scheme, Project No. 262644. S.S. was supported by the National Natural Science Foundation of China under Grant No. 11750110430.
[1] X. Wu and Y. Zhu, Heterogeneous materials: a new class of materials with unprecedented mechanical properties, Mater. Res. Lett. 5, 527 (2017).

[2] P. J. M. Monteiro, P. R. L. Helene, and S. H. Kang, Designing concrete mixtures for strength, elastic modulus and fracture energy, Mater. Struct. 26, 443 (1993).

[3] N. Liang, J. Dai, X. Liu, and Z. Zhong, Experimental study on the fracture toughness of concrete reinforced with multi-size polypropylene fibres, Mag. Concr. Res. 71, 468 (2019).

[4] K. L. Kepple, G. P. Sanborn, P. A. Lacasse, K. M. Gruenberg, and W. J. Ready, Improved fracture toughness of carbon fiber composite functionalized with multi walled carbon nanotubes, Carbon 46, 2026 (2008).

[5] S. M. Lee, Handbook of Composite Reinforcements (Wiley, New York, 1992).

[6] P. K. Mallick, Fiber-Reinforced Composites: Materials, Manufacturing and Design, 2nd ed. (CRC, Boca Raton, FL, 1993).

[7] H. J. Herrmann and S. Roux, Statistical Models for the Fracture of Disordered Media (Elsevier, Amsterdam, 2014).

[8] L. de Arcangelis and H. J. Herrmann, and S. Redner, A random fuse model for breaking processes, J. Phys. Lett. (France) 46, 585 (1985). 
[9] P. M. Duxbury, P. D. Beale, and P. L. Leath, Size Effects of Electrical Breakdown in Quenched Random Media, Phys. Rev. Lett. 57, 1052 (1986).

[10] B. Kahng, G. G. Batrouni, S. Redner, L. de Arcangelis, and H. J. Herrmann, Electrical breakdown in a fuse network with random, continuously distributed breaking strengths, Phys. Rev. B 37, 7625 (1988).

[11] A. Hansen, S. Roux, and H. J. Herrmann, Rupture of centralforce lattices, J. Phys. (France), 50, 733 (1989).

[12] H. J. Herrmann, A. Hansen, and S. Roux, Fracture of disordered, elastic lattices in two dimensions, Phys. Rev. B 39, 637 (1989).

[13] B. Skjetne and A. Hansen, Implications of Realistic Fracture Criteria on Crack Morphology, Front. Phys. 7, 50 (2019).

[14] S. Roux and A. Hansen, Early Stages of Rupture of Disordered Materials, Europhys. Lett. 11, 37 (1990).

[15] S. Roux, A. Hansen, H. J. Herrmann, and E. Guyon, Rupture of heterogeneous media in the limit of infinite disorder, J. Stat. Phys. 52, 237 (1988).

[16] A. A. Moreira, C. L. N. Oliveira, A. Hansen, N. A. M. Araújo, H. J. Herrmann, and J. S. Andrade, Jr., Fracturing Highly Disordered Materials, Phys. Rev. Lett. 109, 255701 (2012).

[17] A. Shekhawat, S. Zapperi, and J. P. Sethna, From Damage Percolation to Crack Nucleation Through Finite Size Criticality, Phys. Rev. Lett. 110, 185505 (2013).

[18] L. de Arcangelis and H. J. Herrrmann, Scaling and multiscaling laws in random fuse networks, Phys. Rev. B 39, 2678 (1989).

[19] L. de Arcangelis, A. Hansen, H. J. Herrmann, and S. Roux, Scaling laws in fracture, Phys. Rev. B 40, 877(R) (1989).

[20] A. Hansen, E. L. Hinrichsen, and S. Roux, Scale-invariant disorder in fracture and related breakdown phenomena, Phys. Rev. B 43, 665 (1991).

[21] S. Pradhan, A. Hansen, and B. K. Chakrabarti, Failure processes in elastic fiber bundles, Rev. Mod. Phys. 82, 499 (2010).

[22] A. Hansen, P. C. Hemmer, and S. Pradhan, The Fiber Bundle Model (Wiley-VCH, Berlin, 2015).

[23] A. Stormo, K. S. Gjerden, and A. Hansen, Onset of localization in heterogeneous interfacial failure, Phys. Rev. E 86, 025101(R) (2012).

[24] L. Furuberg, J. Feder, A. Aharony, and T. Jøssang, Dynamics of Invasion Percolation, Phys. Rev. Lett. 61, 2117 (1988).

[25] S. Roux and E. Guyon, Temporal development of invasion percolation, J. Phys. A 22, 3693 (1989).

[26] S. Maslov, Time Directed Avalanches in Invasion Models, Phys. Rev. Lett. 74, 562 (1995).

[27] J. F. Gouyet, Invasion noise during drainage in porous media, Physica A 168, 581 (1990).

[28] F. T. Peirce, 32X. Tensile Tests for Cotton Yarns v. The Weakest Link Theorems on the Strength of Long and of Composite Specimens, J. Text. Ind. 17, T355 (1926).

[29] H. E. Daniels, The statistical theory of the strength of bundles of threads. I, Proc. R. Soc. London, Ser. A 183, 405 (1945).

[30] D. G. Harlow and S. L. Phoenix, The Chain-of-Bundles Probability Model for the Strength of Fibrous Materials II: A
Numerical Study of Convergence, J. Compos. Mater. 12, 314 (1978).

[31] D. G. Harlow and S. L. Phoenix, Approximations for the strength distribution and size effect in an idealized lattice model of material breakdown, J. Mech. Phys. Solids 39, 173 (1991).

[32] D. Sornette, Mean-field solution of a block-spring model of earthquakes, J. Phys. I (France) 2, 2089 (1992).

[33] P. C. Hemmer and A. Hansen, The Distribution of Simultaneous Fiber Failures in Fiber Bundles, J. Appl. Mech. 59, 909 (1992).

[34] A. Hansen and P. C. Hemmer, Burst avalanches in bundles of fibers: Local versus global load-sharing, Phys. Lett. A 184, 394 (1994).

[35] S. D. Zhang and E. J. Ding, Burst-size distribution in fiberbundles with local load-sharing, Phys. Lett. A 193, 425 (1994).

[36] M. Kloster, A. Hansen, and P. C. Hemmer, Burst avalanches in solvable models of fibrous materials, Phys. Rev. E 56, 2615 (1997).

[37] J. V. Andersen, D. Sornette, and K. T. Leung, Tricritical Behavior in Rupture Induced by Disorder, Phys. Rev. Lett. 78, 2140 (1997).

[38] T. Grossman and A. Aharony, Accessible external perimeters of percolation clusters, J. Phys. A 20, L1193 (1987).

[39] S. Sinha, J. T. Kjellstadli, and A. Hansen, Local load-sharing fiber bundle model in higher dimensions, Phys. Rev. E 92, 020401 (2015).

[40] A. Hansen, The three extreme value distributions: An introductory review, arXiv:2009.03711.

[41] D. Wilkinson and J. F. Willemsen, Invasion percolation: a new form of percolation theory, J. Phys. A 16, 3365 (1983).

[42] B. Nienhuis, in Phase Transitions and Critical Phenomena, edited by C. Domb, M. Green, and J. L. Levowitz (Academic, London, 1987), Vol. 11.

[43] J. L. Cardy, in Phase Transitions and Critical Phenomena, edited by C. Domb, M. Green, and J. L. Levowitz (Academic, London, 1987), Vol. 11.

[44] L. Onsager, Crystal statistics. I. A two-dimensional model with an order-disorder transition, Phys. Rev. 65, 117 (1944).

[45] M. S. S. Challa, D. P. Landau, and K. Binder, Finite-size effects at temperature-driven first-order transitions, Phys. Rev. B 34, 1841 (1986).

[46] R. Lenormand and C. Zarcone, Invasion Percolation in an Etched Network: Measurement of a Fractal Dimension, Phys. Rev. Lett. 54, 2226 (1985).

[47] M. Moura, K. J. Måløy, E. G. Flekkøy, and R. Toussaint, Invasion Percolation in an Etched Network: Measurement of a Fractal Dimension, Phys. Rev. Lett. 119, 154503 (2017).

[48] J. Feder, Fractals (Plenum, New York/London, 1988).

[49] B. Sapoval, M. Rosso, and J. F. Gouyet, The fractal nature of a diffusion front and the relation to percolation, J. Phys. Lett. 46, 149 (1985).

[50] M. Rosso, J. F. Gouyet, and B. Sapoval, Gradient Percolation in Three Dimensions and Relation to Diffusion Fronts, Phys. Rev. Lett. 57, 3195 (1986). 